\title{
Bodenkundlich-morphologische Untersuchungen an einem Buckelwiesenvorkommen im Mangfallgebirge
}

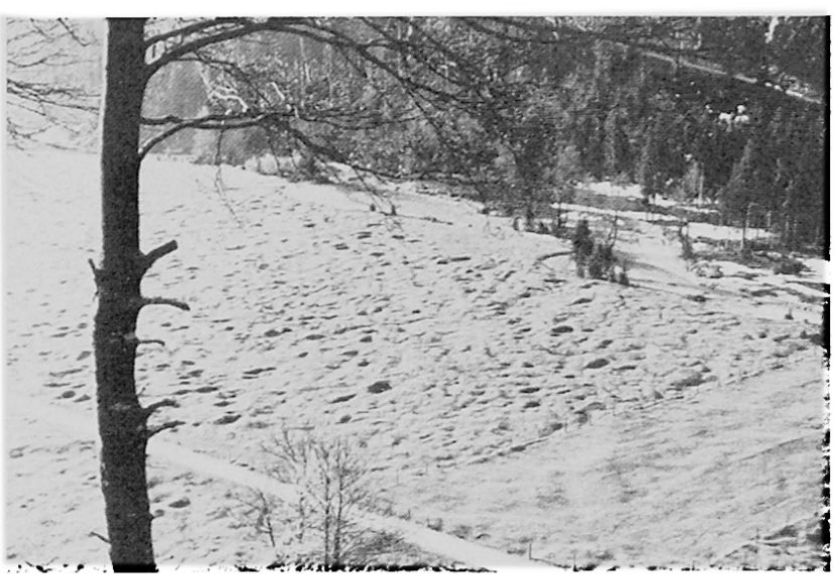

Die Buckelwiesen des Kloaschautales. Die umzäunte Fläche am rechten Bildrand wurde eingeebnet und wird im Gegensatz zu den Buckelwiesen gemäht. Im Hintergrund der Berggraben mit Hochwasserführung.

Redaktionelle Vorbemerkung: Seit mehreren Jahren befaßt sich das Geographische Institut der Universität Zürich mit der Untersuchung von fossilen Böden, die mit verschiedenartigen kaltzeitlichen Schuttmassen wechsellagern. Dabei besteht die Möglichkeit, aus dieser Wechsellagerung Rückschlüsse auf die postglaziale Morphogenese zu ziehen. Der nachfolgende Beitrag von U. Wölfel vermag in diesem Zusammenhang sehr wertvolle Informationen zu vermitteln.

\section{Einleitung}

Eine der interessantesten Kleinformen des Alpenraumes sind die Buckelwiesen. Die dicht gescharten Buckel und Eintiefungen finden sich auf ebenem und geneigtem Gelände unterschiedlichen Substrats sowohl auf bewaldeten als auch auf unbewaldeten Flächen. Die meisten Buckelwiesen wurden auf kalkreichem Lockermaterial gefunden.

Schon seit langem ist ihre Genese ein Gegenstand geomorphologischer Untersuchungen. Viele der seither zu diesem Problem entstandenen Arbeiten basieren auf Verbreitungsuntersuchungen, detaillierte Analysen liegen kaum vor. Eine fundierte Aussage über die Bildungsvorgänge bedarf quantitativer Untersuchungen an Buckelwiesen unterschiedlicher Lage und verschiedener methodischer Ansätze. Die vorliegende Arbeit sollte einen Beitrag hierzu liefern.
Im Laufe der Forschungsgeschichte der Buckelwiesen wurde eine Vielzahl von Theorien zur Genese dieser Kleinform aufgestellt (ausführliche Zusammenstellung bei w. ENGELSCHALK 1971). Heute werden nur noch zwei diskutiert: die Frosttheorie und die Kalklösungstheorie.

Die Frosttheorie (u. a. vertreten von J. L. LUTZ und H. PAUL 1947, E. SCHÖNHALS 1957, W. ENGELSCHALK 1971) geht davon aus, daß die Buckelwiesen «im Übergang von der letzten Eiszeit zur gegenwärtigen Warmzeit» (W. ENGELSCHALK 1971, S. 144) entstanden sind, das heißt daß die Buckelwiesen eine fossile Form sind. Gemäß der Frosttheorie sind die Buckel die aktiven Formen und wurden durch den Druck von Eiskeilen bzw. Eisrahmennetzen herausgepreßt und durch Frosthebung und Solifluktion teilweise umgestaltet.

Die Vertreter der Kalklösungstheorie (u. a. E. EBERS 1959, S. MÜLLER 1959, Z. GRACANIN 1970) betrachten die Buckelwiesenbildung als einen noch heute andauernden Vorgang: «... nicht das Herausheben der Buckel, sondern die Bildung der Dellen ist entscheidend, die durch Lösungsverwitterung und Bodenbildung im carbonatischen Schutt im Laufe langer Perioden erfolgt» (Z. GRACANIN 1970, S. 203).

Die Überprüfung dieser beiden Theorien war der Ausgangspunkt für die Untersuchung der Kloaschauer Buckelwiesen.

\section{Beobachtungen}

\section{Äußere Form (Fig. 1 und Abbildung)}

Die Buckelwiesen des Kloaschautales (ca. $900 \mathrm{~m}$ über NN) sind auf dolomitischem Talschutt ausgebildet (Gefälle 3\%). Die zum größten Teil länglich-ovalen Buckel weisen einen Durchmesser von 1-3 m auf und liegen in unregelmäßigen Abständen; die Buckellängsachsen bevorzugen keine bestimmte Himmelsrichtung. Die Eintiefungen zwischen den Buckeln sind in der Mehrzahl geschlossene Hohlformen. Die Dichte der Buckel pro Hektar beträgt ca. 500. Die größten Höhendifferenzen zwischen Buckelscheiteln und Eintiefungen erreichen über $1 \mathrm{~m}$. Auffallend ist der Vegetationsunterschied von Buckeln und Eintiefungen: eine dürre

Ursula Wölfel, Studienreferendarin, Ludwig-Weis-Strasse 10, D-87 Würzburg 


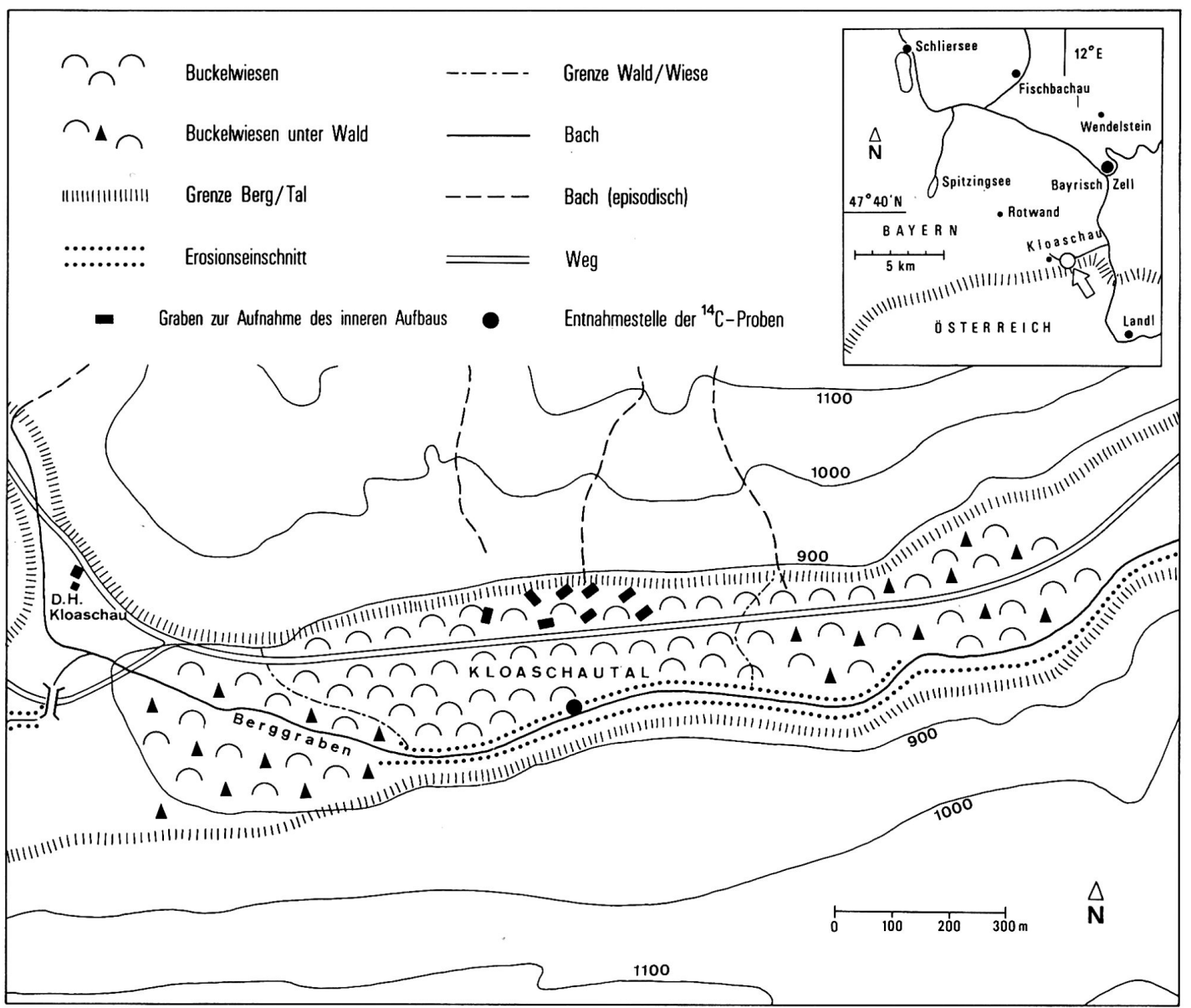


schüttere Vegetation auf den Buckeln zeigt Trockenheit an, während in den Eintiefungen eine dichte Wiesenvegetation auf größere Feuchtigkeit schließen läßt.

\section{Innerer Aufbau}

Um den inneren Aufbau der Buckel und Eintiefungen studieren zu können, wurden mehrere Gräben ausgehoben und die Schichten aufgenommen. Figur 2 zeigt ein Beispiel dieser Aufschlüsse. In den Eintiefungen finden sich gut entwickelte Rendzinen mit mächtigen A-Horizonten und taschenförmig in den Schutt eingreifenden $\mathrm{C}_{\mathrm{v}}$-Horizonten. Die im $\mathrm{C}_{\mathrm{v}}$-Horizont enthaltenen Steine sind größtenteils verascht. Unter den Buckeln ist der $\mathrm{C}_{\mathrm{v}}$-Horizont in der Regel weniger mächtig und nur leicht verwittert. Der A-Horizont ist selten über $10 \mathrm{~cm}$ stark und enthält einen nicht unbedeutenden Anteil Steine.

Der kantige bis kantengerundete Schutt (linsenförmige Horizontalschüttung) ist ungestört, das heißt es zeigen sich keine Aufwölbungen oder Pressungsstrukturen. Die Schichten verlaufen sowohl unter den Buckeln als auch unter den Eintiefungen etwa horizontal. An den Flanken der Buckel ist zu beobachten, daß einige Schichten zu den Eintiefungen hin abbiegen. Eine Materialsortierung, wie sie nach der Frosttherapie möglich ist, zeigt sich nicht.

\section{Untersuchungen}

\section{Bodenbohrungen}

Um zu überprüfen, ob die Boden- und Verwitterungsmächtigkeit in den Eintiefungen erheblich größer als unter den Buckeln ist, - ein Indiz für die Kalklösungstheorie - wurden zahlreiche Bodenbohrungen durchgeführt. Das Verhältnis der Mächtigkeiten der Bodenhorizonte von Buckeln und Eintiefungen beträgt durchschnittlich $1: 7$.

\section{Dünnschliffuntersuchungen}

Mit diesen Untersuchungen sollte der Frage nachgegangen werden, ob das in den Aufschlüssen beobachtete Abbiegen von Schichten zu den Eintiefungen hin auf Frostpressung oder auf Grund von Lösungsvorgängen in den Eintiefungen zurückzuführen ist.

Dazu wurden aus einer zur Eintiefung abbiegenden Schicht, aus einer horizontal verlaufenden Schicht und aus dem $\mathrm{C}_{\mathrm{v}}$-Horizont derselben Eintiefung Dünnschliffe angefertigt (Orientierung nach der Horizontalen), um etwaige Strukturunterschiede feststellen zu können.

Bei keinem der Schliffe konnten bei der ersten Betrachtung unter dem Mikroskop Besonderheiten in der Struktur festgestellt werden. Da jedoch Feinheiten der Struktur bei bloßer Betrachtung nicht immer in Erscheinung treten, wurden Einregelungsmessungen der in den Proben enthaltenen Feinsande vorgenommen. Es zeigt sich, daß die Längsachsen weder in der horizontal verlaufenden noch in der zur Eintiefung abbiegenden Schicht eine besondere Richtung bevorzugen. Die Feinsande im $\mathrm{C}_{\mathrm{v}}$-Horizont, die als Vergleich dienten, sind zu 20\% horizontal und zu $40 \%$ vertikal gelagert, das heißt abweichend von der Gleichverteilung.

Ein Einfluß von Frostpressung auf die Schichten der Buckelflanken kann aus dieser Untersuchung nicht erschlossen werden.

\section{Einregelungsmessungen}

Frosteinwirkung kann sich in Veränderungen der ursprünglichen Lagerung der Steine dokumentieren. Dies gilt besonders für die von der Frosttheorie vertretene nachträgliche Umgestaltung der Buckel durch Frosthebung.

Einregelungsmessungen in verschiedenen Bereichen von Buckeln ergaben keine Abweichung von der im Bachbett ermittelten Normallagerung der Steine.

\section{Laboruntersuchungen}

Für einen Vergleich der physikalischen und chemischen Zusammensetzung in einem Buckelprofil mit den entsprechenden Verhältnissen im Profil unter einer Eintiefung wurden, ausgehend von der Bodenoberfläche, in 10-cm-Abständen jeweils 10 Proben entnommen und deren Kalkgehalt, Korngrößenzusammensetzung, Bodenazidität, Kohlenstoff- und Stickstoffgehalte bestimmt:

\section{Kalkgehalt (nach SCHEIBLER)}

Im Buckel sind lediglich die obersten $10 \mathrm{~cm}$ in bedeutenderem Maße $(40 \%)$ entkalkt. Bereits in einer Tiefe von $10-20 \mathrm{~cm}$ steigt der Karbonatgehalt auf $81 \%$ an und erreicht in den Tiefenzonen darunter Werte von 93-98\%. 


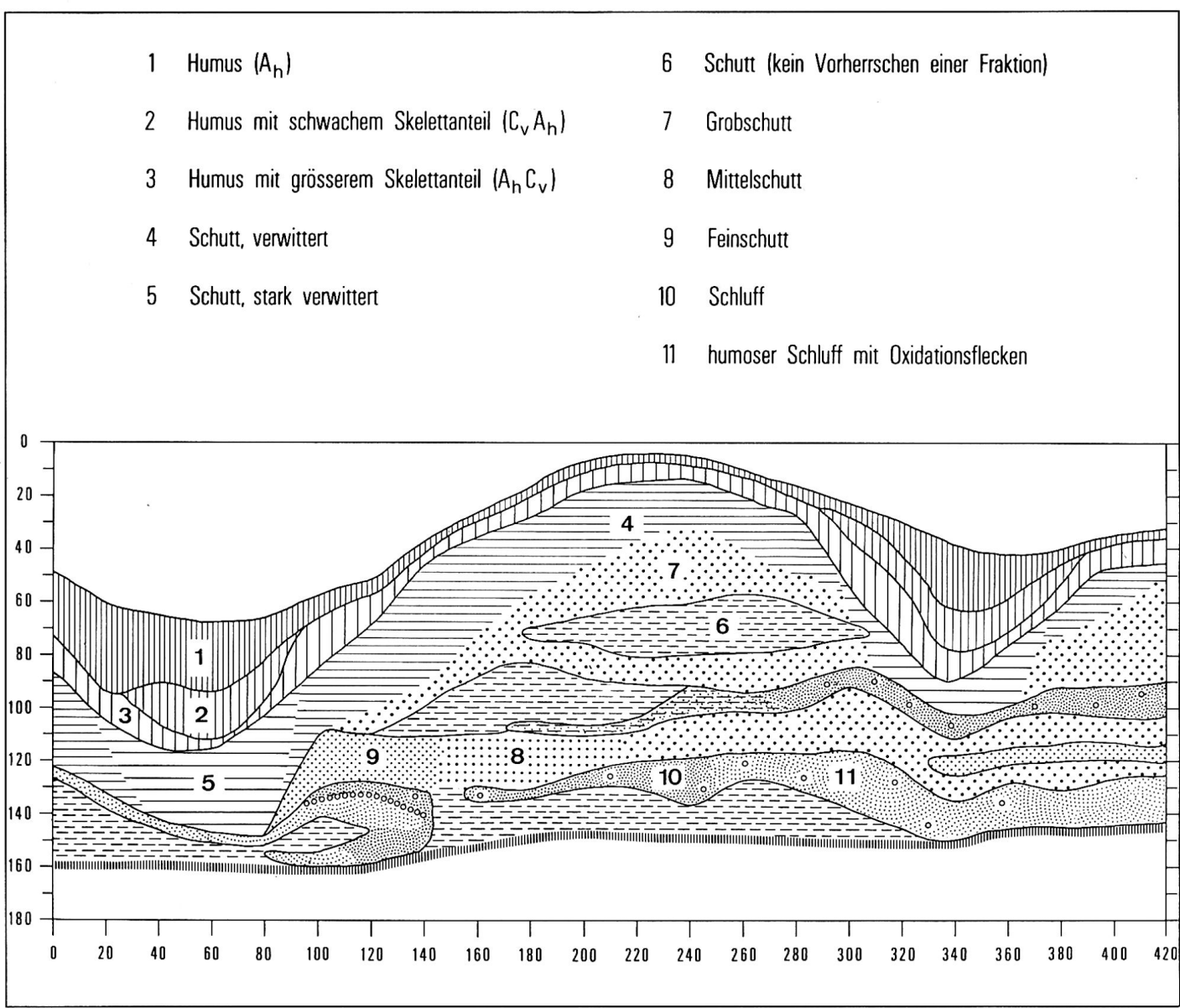


Ein völlig anderes Bild zeigt das Profil der Eintiefung. Die oberen $30 \mathrm{~cm}$ sind fast völlig karbonatfrei. Im Tiefenbereich 40-50 cm erfolgt ein sprunghaftes Ansteigen des Kalkgehaltes auf $85 \%$, darunter ein langsames Anwachsen auf 95\%. Der Karbonatgehalt ist in $1 \mathrm{~m}$ Tiefe des Profils jedoch immer noch geringer als der im entsprechenden Bereich des Buckels. Für die Eintiefung ist damit eine bis in den C-Horizont reichende Entkalkung nachgewiesen.

Das Ergebnis der Kalkgehaltsbestimmung unterstützt die Kalklösungstheorie. Es sei angemerkt, daß Z. GRACANIN (1970) ähnliche Ergebnisse bei der Untersuchung der Lechtaler Buckelwiesen erzielte.

Korngrößen (Siebung und Aräometerbestimmung, Ergebnisse in Fig. 3).

Bei der Gesamtkorngrößenanalyse zeigt sich, daß im Buckel lediglich im A-Horizont $(0-10 \mathrm{~cm})$ ein größerer Feinerdegehalt $(32 \%)$ vorhanden ist, während im Profil der Eintiefung der Feinerdeanteil bis in die Tiefe von $50 \mathrm{~cm}$ dominiert; der A-Horizont $(0-30 \mathrm{~cm})$ besteht zu $90 \%$ aus Feinerde. Erst in den Tiefenbereichen von $80-100 \mathrm{~cm}$ ist die Gesamtkorngrößenzusammensetzung des Buckels mit der der Eintiefung vergleichbar.

Auch die Textur der Feinerde zeigt einen deutlichen Unterschied zwischen Buckel und Eintiefung. Im Buckelprofil überwiegt die Sandfraktion. Sie erreicht im A-Horizont etwa 10\%, nimmt dann mit der Tiefe auf $60 \%$ zu. Der Schluffanteil dominiert in den oberen $20 \mathrm{~cm}$ und verringert sich dann zugunsten des Sandanteils auf durchschnittlich $32 \%$. Besonderes Augenmerk sollte auf den Tonanteil gelegt werden: nur im A-Horizont ist er etwas größer (15\%), im übrigen Profil liegt sein Anteil bei durchschnittlich $8 \%$.

In der Eintiefung erreicht der Tonanteil im A-Horizont einen Prozentsatz von 36; allmählich nimmt er ab und stellt sich erst ab $40 \mathrm{~cm}$ Tiefe auf den Wert von $8 \%$ ein, wie er im C-Horizont des Buckels vorhanden ist. Die Feinerde der Eintiefung wird mit einem Prozentsatz zwischen 47 und 65 vom Schluff dominiert. Der Sandanteil nimmt nach unten von 10 auf $40 \%$ zu, erreicht aber auch in den tiefen Bereichen nicht den großen Anteil wie im Buckelprofil.

Besonders die Feinerdeanalysen lassen die unterschiedliche Verwitterungsdynamik von Buckel und Eintiefung erkennen. Die Zersetzung der anorganischen Bestandteile des A-Horizontes der Eintiefung ist weit vorangeschritten; nur noch wenige Prozent Sand sind enthalten und der Dolomit hat sich aufgelöst, wobei sich Residualton anreicherte. Im Buckel hingegen ist die Tonbildung noch im Anfangsstadium; der A-Horizont weist nur wenige Prozent mehr Ton auf als das von der Verwitterung nicht erreichte Material in tieferen Lagen. Es ergibt sich somit eine Übereinstimmung der Korngrößenanalysen mit den Kalkgehaltsbestimmungen: beide Untersuchungen zeigen für den Buckel eine geringe, für die Eintiefung eine hohe Verwitterungsintensität.

\section{Bodenazidität (gemessen in 0,1 n-KCl-Lösung)}

Die Bodenreaktion stellt sich in etwa analog zu den Kalkgehaltsmessungen dar. Der Buckel zeigt nur im A-Horizont eine schwach saure Reaktion (pH 6,9), während im übrigen Profil eine schwach alkalische Reaktion vorliegt ( $\mathrm{pH} 7,3-7,9$ ). In der Eintiefung ist bis in die Tiefe von $40 \mathrm{~cm}$ eine mäßig bis schwach saure Reaktion ( $\mathrm{pH} 5,8-6,8)$ zu verzeichnen. Im Tiefenbereich ab $50 \mathrm{~cm}$ liegen die pH-Werte bei 7,6-7,7, das heißt daß auch die Bodenazidität im C-Horizont der Eintiefung höher ist als die des Buckels im entsprechenden Bereich.

Organische Substanz (Kohlenstoff und Stickstoff) Der Kohlenstoffgehalt (Bestimmung durch nasse Oxidation) ist im A-Horizont der Eintiefung ungefähr doppelt so hoch wie im A-Horizont des Buckels (43\% : $19 \%)$.

Die Stickstoffbestimmungen spiegeln die Werte der Kohlenstoffgehalte wieder.

Auch hieraus folgt, daß die Bodenentwicklung der Eintiefung weiter fortgeschritten ist als die des Buckels.

\section{${ }^{14} \mathrm{C}$-Datierungen}

Die Buckelwiesen im Kloaschautal waren nicht zuletzt deswegen als Untersuchungsobjekt ausgewählt worden, weil sich die Möglichkeit ergab, das Alter dieser Buckelwiesen zu bestimmen.

Durch den Erosionseinschnitt des Baches (Berggraben) sind die oberen $3 \mathrm{~m}$ der Talverfüllung aufgeschlossen. Vier übereinanderliegende, gewellte, schluffig-humose Horizonte durchziehen annähernd horizontal den Schuttkörper. Diese gewellten Horizonte entsprechen dem Bild, das der Boden der aktuellen 
Fig. 3:

Oben links: Gesamtkorngrößenverteilung im Profil eines Buckels.

Unten links: Korngrößenverteilung in der Feinerde eines Buckels.
Oben rechts: Gesamtkorngrößenverteilung im Profil einer Delle.

Unten rechts: Korngrößenverteilung in der Feinerde einer Delle.
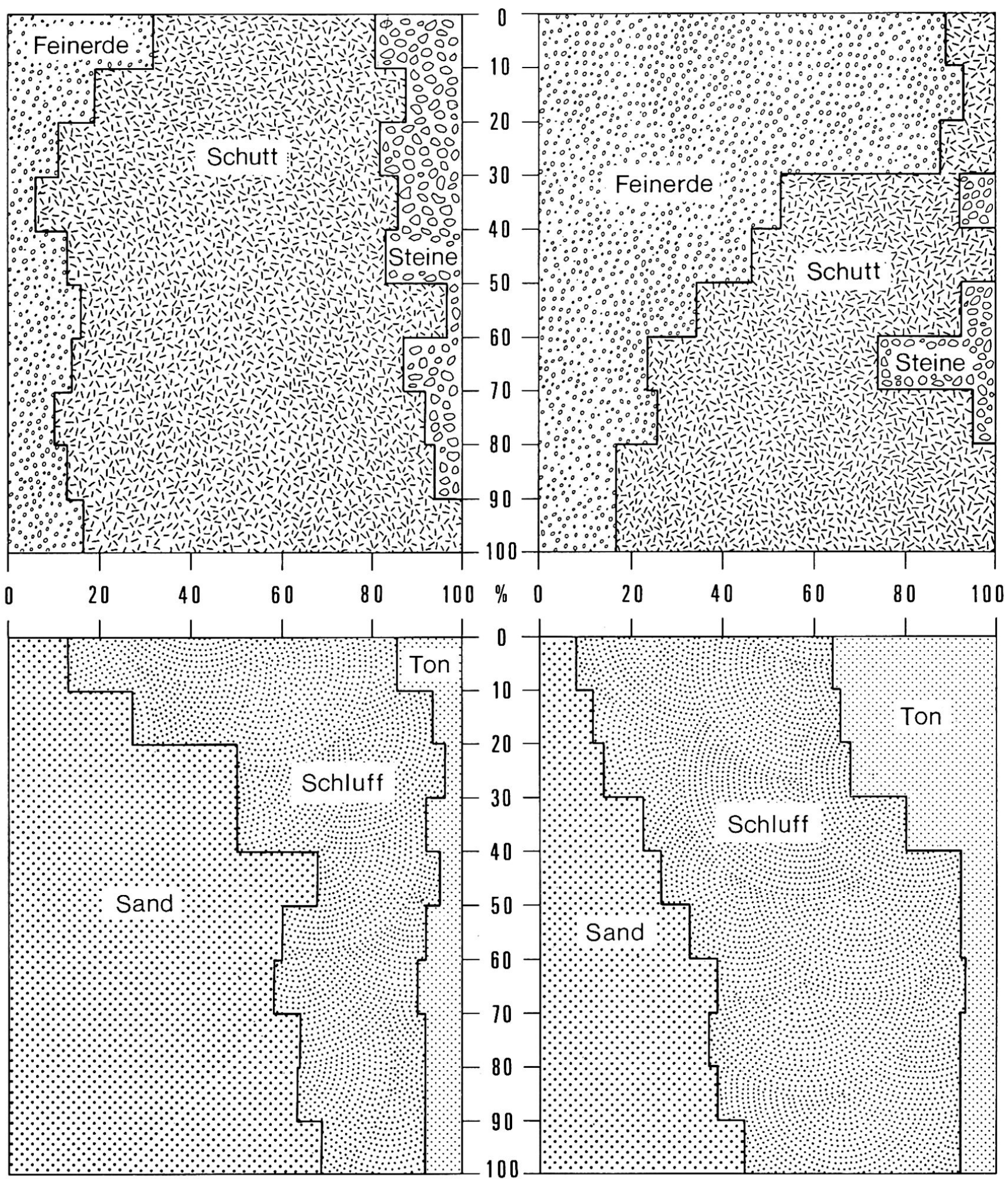
Buckel und Eintiefungen im Längsschnitt bietet. Es handelt sich um verschüttete ältere Taloberflächen.

Die Datierung dieser Horizonte ergab für den untersten, in 2,5 m Tiefe liegenden Horizont ein Alter von $2245 \pm 80$ Jahren, für den rezenten Horizont (die Probe wurde aus der tiefsten Stelle einer Eintiefung entnommen) ein Alter von $955 \pm 35$ Jahren.

Für die Genese der Kloaschauer Buckelwiesen folgt aus den Datierungen, daß diese Buckelwiesen rezent gebildet wurden und die Bildung in relativ kurzer Zeit vor sich gegangen ist. Selbst wenn zu den durch die ${ }^{14} \mathrm{C}$-Datierung erhaltenen Mindestaltern noch eine mehr oder weniger lange Periode addiert werden müßte (Zeit zwischen Überschüttung und Bildung des entnommenen Humus), ändert sich nichts am grundlegenden Ergebnis: die Buckelwiesen des Kloaschautales haben sich im jüngeren Holozän gebildet.

\section{Zur Genese der Kloaschauer Buckelwiesen}

Wie aus den Untersuchungen hervorgeht, sind für die Kloaschauer Buckelwiesen keinerlei Anzeichen für eine Frostprägung vorhanden, sondern die Ergebnisse weisen auf Kalklösungsvorgänge hin.

Wie jedoch kommt es zur Ausbildung von Buckelwiesen? Der Schutt des Kloaschautales ist nicht homogen, sondern besteht aus Schichten und Linsen unterschiedlicher Korngrößenzusammensetzungen. Die Oberfläche des frisch abgelagerten Schuttes ist durch kleine Rinnen und Kolke mit zumeist feinen Korngrößen gegliedert. Diese Inhomogenitäten bewirken eine karstmäßige Sickerwasserführung. Das Niederschlagswasser versitzt an Stellen mit geringem Feinerdematerialanteil relativ rasch und löst dabei Karbonate. Jedoch ist diese Strömungsanlösung weniger effektiv als die Durchdringungsverwitterung (H. ROHDENBURG und B. MEYER 1963), die an Stellen mit dichter lagerndem Schuttmaterial stattfindet. Da die feinerdereichen Partien hauptsächlich in den Vertiefungen vorkommen, muß berücksichtigt werden, daß sowohl von umliegenden höheren Schuttakkumulationen als auch durch in den Rinnen fließendes Wasser das Wasserangebot in den Vertiefungen vermehrt wird. Die größtmögliche Kalklösung erfahren also Vertiefungen mit hohem Feinerdegehalt. Entsprechend der Inhomogenität des Schuttes greifen die Entkalkungsfronten unregelmäßig tief ein.
In der Phase der Vegetationsansiedlung und der Initialbodenbildung verstärkt sich die flächenmäßige Differenzierung der Kalklösung. Bodenbildung und Pflanzenansiedlung geschieht auf Grund der Feuchtigkeitsverhältnisse und des Feinerdegehalts bevorzugt an den Stellen mit intensiver Kalklösung. Das durch die Huminsäuren angereicherte Wasser ist kalkaggressiver, wodurch die Lösungstätigkeit in diesen Bereichen verstärkt wird. In diesem Stadium wird zum größten Teil festgelegt, an welchen Stellen die Eintiefungen der Buckelwiesen liegen und welche Schuttpartien stehenbleiben bzw. nur geringfügig erniedrigt werden.

Es ist nicht auszuschließen, daß außer der Vorstrukturierung durch die besondere Art der Schuttakkumulation noch andere Umstände auf die Entwicklung des Buckel-Eintiefungs-Mosaiks eingewirkt haben, wie z. B. Setzungserscheinungen im Schutt, Humuseinschlüsse in der Schuttmasse oder die Baumvegetation, auf deren Wirkung bereits S. MÜLLER (1959) hingewiesen hat. Bei weiter fortgeschrittenem Stadium der Buckelwiesenbildung kann hinzutreten, daß Bodenmaterial, insbesondere Humus, von den Buckeln in die Eintiefungen geschwemmt wird, was die Bodenentwicklung auf den Buckeln hemmt und das Huminsäureangebot in den Eintiefungen vergrößert.

Wie z. B. auf den Buckelwiesen im Lechtal beobachtet werden kann, gehen die Eintiefungen von Buckelwiesen bei langer Entwicklungsdauer (Z. GRACANIN 1970) zum Teil in Dolinen über. Damit ist das Ende der Buckelwiesenentwicklung erreicht. Im Kloaschautal befinden sich die Buckelwiesen jedoch noch nicht in diesem Stadium.

\section{Zusammenfassung}

Über die Entstehung der Buckelwiesen bestehen zwei gegensätzliche Theorien: die Frosttheorie und die Kalklösungstheorie. Die Untersuchungen an den Buckelwiesen im Kloaschautal (Bayrische Kalkalpen) erbrachten, daß diese Buckelwiesen nicht durch Frosteinwirkung entstanden sind. Ihre Entwicklung beruht auf einer durch fluviatile Ablagerung geschaffenen Vorstrukturierung des Ausgangsmaterials und der sich darauf vollziehenden flächenmäßig differenzierten Kalklösung. 


\section{Literatur:}

EBERS, E.: Die Buckelwiesen: nicht Eiszeitalter, sondern Gegenwart. Eiszeitalter und Gegenwart, Bd. 10, 1959

ENGELSCHALK, W.: Alpine Buckelfluren. Untersuchungen zur Frage der Buckelwiesen im Bereich des eiszeitlichen Isargletschers. Regensburger Geogr. H., Bd. 1, 1971

GRACANIN, Z:: Buckelwiesen und ihre Bodenbildung in den westlichen Lechtaler Alpen. Allg. Forst- und Jagdztg., Bd. 141, H. 10, 1970

LUTZ, J. und PAUL, H.: Die Buckelwiesen bei Mittenwald. Ber. d. Bayr. Bot. Ges. zur Erforschung der heimischen Flora, Bd. 27, 1947

MÜLLER S.: Buckelwiesen, Beobachtungen aus den Julischen Alpen. Kosmos, Jg. 55, 1959

ROHDENBURG, H. und MEYER,B.: Rezente Mikroformung in Kalkgebieten durch inneren Abtrag und die Rolle der periglazialen Gesteinsverwitterung. Z. f. Geom., N. F., Bd. 7, 1963

SCHÖNHALS, E.: Späteiszeitliche Windablagerungen in den nördlichen Kalkalpen und die Entstehung der Buckelwiesen. Natur und Volk, Bd. 87, 1957 